(FNA) of the pancreas requires experienced cytopathologists as well as advanced immunohistochemical assays to obtain a final diagnosis on a small amount of tissue. A 46-year-old man complaining of watery diarrhea and severe weight loss (more than $20 \mathrm{~kg}$ ) for more than 1 year was admitted to our hospital due to severe diabetic crisis. Enlarged lymph nodes $(2.5 \times 1 \mathrm{~cm})$ were found at the right axillary stations. Abdominal ultrasound revealed the presence of a large hyperechogenic mass, mainly located at the pancreatic head. Abdominal computed tomography scan confirmed a diffuse enlargement of the head and body of the pancreas associated with lymphadenopathy along the lesser gastric curvature. Percutaneous ultrasound-guided FNA of the pancreas as well as gross biopsy of the axillary lymph nodes were unable to identify the nature of the mass. Diagnostic laparoscopy was performed: several enlarged lymph nodes along the lesser gastric curvature were revealed. Multiple biopsies of the pancreatic head were taken and lymphadenectomy along the lesser curvature and the hepatic hilus was also performed. The definitive histopathological examination of the pancreatic specimen revealed a primary low-grade non-Hodgkin B cell pancreatic lymphoma. The postoperative course was unremarkable; the patient underwent systemic chemotherapy regime for low-grade B cell Hodgkin lymphoma and he was symptom free at 9-month follow-up.

Key words: Diagnostic laparoscopy - Pancreatic lymphoma

Correspondence to: L. Boni

\section{Laparoscopic renal biopsy in bilateral pelvic kidney with chronic glomerulonephritis}

\author{
Y. Bayazit, ${ }^{1}$ I. A. Arídoğan, ${ }^{1}$ S. Özdemir, ${ }^{1}$ I. Karayaylali, ${ }^{2}$ S. \\ Doran $^{1}$ \\ ${ }^{1}$ Faculty of Medicine, Department of Urology, Çukurova University, \\ Balcali, 01330 Adana, Turkey \\ ${ }^{2}$ Faculty of Medicine, Department of Nephrology, Çukurova Uni- \\ versity, Balcali, 01330 Adana, Turkey \\ Received: 11 January 2001/Accepted in final form: 26 November 2001/ \\ Online publication: 3 May 2002 \\ DOI: $10.1007 / \mathrm{s} 00464-001-4243-5$
}

\section{Abstract}

Percutaneous needle biopsy under the guidance of ultrasound or computerized tomography is the most valuable method in the diagnosis of parenchymal kidney diseases. However, sometimes it can be difficult to perform in the presence of certain anomalies, anatomic variations, or medical problems. In the presence of bilateral pelvic kidney, which is a rare anomaly, laparoscopy can be used to obtain biopsy. Biopsy of kidney was planned in a 26-year-old woman who presented with a history of hypertension for 7 years and proteinuria with the diagnosis of nephrotic syndrome. For the biopsy, the laparoscopic approach was chosen since the patient had bilateral pelvic kidneys. Under general anesthesia, using three port sites, the right kidney was reached, which was located more anterior than the left one. Three biopsy specimens for histologic evaluation were taken with a Tru-Cut biopsy needle. No complications were en- countered during or after the operation. The patient was ceased from urological follow-up after performing an ultrasound on the first postoperative day. This is the first case of pelvic kidney with chronic glomerulonephritis reported in the literature in which the histologic diagnosis was made with the help of laparoscopy. Laparoscopic kidney biopsy is a minimally invasive technique that can be done in cases with anatomic variations, making percutaneous needle biopsy impossible.

Key words: Pelvic kidney - Ectopia - Laparoscopy Biopsy - Glomerulonephritis - Parenchymal disease Correspondence to: Y. Bayazit

\section{Early small bowel obstruction complicating hand-assisted laparoscopic donor nephrectomy}

\section{An uncommon etiology}

R. L. Madden, A. Kurbanov, B. J. Benedetto, G. S. Lipkowitz Department of Surgery, Baystate Medical Center, West Springfield, MA 01199, USA and Tufts University School of Medicine,

Boston, MA 02111, USA

Received: 18 October 2001/Accepted in final form: 28 November 2001/Online publication: 3 May 2002

DOI: $10.1007 / \mathrm{s} 00464-001-4142-9$

\section{Abstract}

Laparoscopic donor nephrectomy is gaining widespread acceptance as a minimally invasive technique for kidney donation. Although it has been associated with decreased patient morbidity and more rapid recovery, it exposes patients to possible complications inherent in its transperitoneal route. We report a case of a small bowel obstruction secondary to midjejunal intussusception occurring on the third postoperative day after a handassisted laparoscopic donor nephrectomy. The intussusception proved to be idiopathic since no lead point was identified. The patient recovered without significant sequela after reduction of the intussusceptum. Postoperative ideopathic intussusception is an uncommon cause of bowel obstruction in adults. Surgeons that perform laparoscopic donor nephrectomy will need to remain vigilant for complications that can be associated with the intraperitoneal route of this technique.

Key words: Hand-assisted laparoscopy — Laparoscopic nephrectomy - Small bowel intussusception Correspondence to: R. L. Madden

\section{Laparoscopic treatment of a gastric outlet obstruction caused by a gallstone (Bouveret's syndrome)}

\author{
P. Malvaux, ${ }^{1}$ R. Degolla, ${ }^{1}$ M. De Saint-Hubert ${ }^{2}$ \\ E. Farchakh, ${ }^{2}$ P. Hauters ${ }^{1}$ \\ ${ }^{1}$ Department of Surgery, Clinique Notre-Dame, Avenue Delmée, 9, \\ B-7500 Tournai, Belgium \\ ${ }^{2}$ Department of Gastroenterology, Clinique Notre-Dame, Avenue \\ Delmée, 9, B-7500 Tournai, Belgium
}

Received: 12 July 2000/Accepted in final form: 15 August 2001/Online publication: 3 May 2002

DOI: $10.1007 / \mathrm{s} 004640042033$ 